PROCEEDINGS OF THE

AMERICAN MATHEMATICAL SOCIETY

Volume 131, Number 9, Pages 2877-2881

S 0002-9939(03)07044-8

Article electronically published on February 28, 2003

\title{
A TAUBERIAN THEOREM FOR VILENKIN SERIES
}

\author{
W. R. WADE
}

(Communicated by Andreas Seeger)

\begin{abstract}
There are a number of papers in the literature which contain Cesàro analogues of results already known for martingale sums of VilenkinFourier series. We show that for Vilenkin systems of bounded type, these are not merely analogues but actually generalizations. Indeed, we prove that convergence of the Cesàro means of a Vilenkin series $S$ implies convergence of martingale partial sums of $S$ itself.
\end{abstract}

\section{$\S 1$ INTRODUCTION}

Let $\mathbf{N}:=\{0,1,2, \cdots\}$, and $\mathcal{P}:=\left\{p_{0}, p_{1}, \cdots\right\}$ be any sequence of integers which satisfies $p_{n} \geq 2$. For each $n \in \mathbf{N}$ set $P_{n}:=p_{0} p_{1} \cdots p_{n-1}$, where the empty product is by definition 1 . The multiplicative Vilenkin group associated with $\mathcal{P}$ is the set $G:=\left\{\left(x_{0}, x_{1}, \cdots\right): x_{k} \in \mathbf{N}\right.$ and $\left.0 \leq x_{k}<p_{k}\right\}$ together with the operation

$$
x \dot{+} y:=\left(x_{0} \oplus y_{0}, x_{1} \oplus y_{1}, \cdots\right),
$$

where $x=\left(x_{0}, x_{1}, \cdots\right), y=\left(y_{0}, y_{1}, \cdots\right)$ and, for each $k, x_{k} \oplus y_{k}$ represents the sum of $x_{k}$ and $y_{k}$ modulo $p_{k}$. The dual group of $G$ is the system $\left(w_{n}, n \in \mathbf{N}\right)$ defined for $x=\left(x_{0}, x_{1}, \cdots\right)$ by

$$
w_{n}(x):=\prod_{k=0}^{\infty} \exp \left(\frac{2 \pi i n_{k} x_{k}}{p_{k}}\right),
$$

where the coefficients $n_{k}$ are integers which satisfy $0 \leq n_{k}<p_{k}$ and $n=\sum_{k=0}^{\infty} n_{k} P_{k}$ (see Vilenkin [4] for details). When $p_{k}:=2$ for all $k$, the group $G$ is called the dyadic group and the characters $w_{n}$ are called the Walsh system. When $p_{k}=O(1)$, the system $\left\{w_{n}\right\}$ is called a (multiplicative) Vilenkin system of bounded type.

It is well known that $G$ is a compact group for each collection of radices $\mathcal{P}$, and that the corresponding Vilenkin system $\left\{w_{n}\right\}$ is a complete orthonormal system on $G$. Moreover, the group $G$ can be identified with the interval $[0,1)$ by taking an $x=\left(x_{0}, x_{1}, \cdots\right) \in G$ to the number

$$
\bar{x}:=\sum_{k=0}^{\infty} x_{k} P_{k+1}^{-1} .
$$

Under this identification, Haar measure on $G$ is taken to Lebesgue measure on $[0,1)$.

Received by the editors April 10, 2002.

2000 Mathematics Subject Classification. Primary 42C10, 43A75.

(C)2003 American Mathematical Society 
A Vilenkin series is a series of the form $S:=\sum_{k=0}^{\infty} a_{k} w_{k}$, where $a_{k}$ is some sequence of complex numbers. For each $x \in G$ and $n \in \mathbf{N}$, the partial sums of a Vilenkin series $S$ are defined by

$$
S_{n}(x):=\sum_{k=0}^{n-1} a_{k} w_{k}(x) .
$$

The partial sums $S_{P_{n}}$ form a martingale in $L^{2}(G)$ which allows one to use martingale convergence theorems on Vilenkin series.

\section{§2. Preliminaries}

For each nonnegative integer $n$, define intervals on $G$ by $I_{0}(0):=G$, and

$$
I_{n}(j):=\left\{x=\left(x_{0}, x_{1}, \cdots\right) \in G: \sum_{k=0}^{n-1} x_{k} P_{k+1}^{-1}=\frac{j}{P_{n}}\right\}
$$

for $j=0,1, \cdots, P_{n}-1, n=1,2, \cdots$. Recall that $\left\{I_{n}(0)\right\}_{n=0}^{\infty}$ is a nested sequence of subgroups of $G$ which forms a neighborhood base at the origin, and for each $n$, $\left\{I_{n}(j)\right\}_{j=0}^{P_{n}-1}$ is a collection of pairwise disjoint compact sets in $G$ whose union is $G$. In particular, given $x \in G$ and $n \in \mathbf{N}$, there is a unique $0 \leq j<P_{n}$ such that $x \in I_{n}(j)$. We shall denote this interval by $I_{n}(x)$.

Denote the Haar measure of a subset $E$ of $G$ by $m(E)$ and the Lebesgue measure of a subset $E$ of $[0,1)$ by $|E|$. Notice that under the identification of $G$ with $[0,1)$, the interval $I_{n}(j)$ corresponds to the interval $\left[j P_{n}^{-1},(j+1) P_{n}^{-1}\right)$. In particular,

$$
m\left(I_{n}(j)\right)=P_{n}^{-1}
$$

for $0 \leq j<P_{n}$ and $n \in \mathbf{N}$.

Let $x \in G$. A sequence of measurable sets $E_{j}$ in $G$ is said to shrink nicely to $x$ if there exist integers $r_{j} \rightarrow 0$, as $j \rightarrow \infty$, and an absolute constant $\alpha>0$ such that $E_{j} \subset I_{r_{j}}(x)$ and

$$
m\left(E_{j}\right) \geq \alpha \cdot m\left(I_{r_{j}}(x)\right)
$$

for $j=1,2, \ldots$ By using the identification of $G$ with the unit interval, and of Haar measure with Lebesgue measure, it is easy to check that if $E$ is a measurable subset of $G$ and $h$ represents the characteristic function of $E$, i.e., $h(x)=1$ for $x \in E$ and $h(x)=0$ for $x \notin E$, then

$$
\frac{1}{m\left(E_{j}\right)} \int_{E_{j}} h d m \rightarrow 1
$$

almost everywhere $[m]$ on $E$ for any sequence of sets $E_{j}$ which shrinks nicely to $x$ (see Rudin [2], p. 140). In particular, if $E_{j}$ shrinks nicely to $x$, then

$$
\lim _{j \rightarrow \infty} \frac{m\left(E \cap E_{j}\right)}{m\left(E_{j}\right)}=1
$$

for almost every $x \in E$. 


\section{§3. The MAIN RESUlt}

For each $x \in G$ and each integer $n>0$, the Cesàro means of $S$ are defined by

$$
\sigma_{n}(x):=\frac{1}{n} \sum_{k=1}^{n} S_{k}(x)
$$

It is well known (and easy to see) that if $S_{k}$ converges to some limit $f$, as $k \rightarrow \infty$, then $\sigma_{n} \rightarrow f$, as $n \rightarrow \infty$. We will obtain the following partial converse to this result. (The Walsh version of this result was obtained by Shaginyan [3] in 1974.)

Theorem 1. Suppose $E$ is a measurable subset of $G$ and $S$ is a Vilenkin series whose Cesàro means satisfy

$$
\lim _{n \rightarrow \infty} \sigma_{P_{n}}(x)=f(x), \quad x \in E,
$$

for some finite-valued, measurable function $f$. If the Vilenkin system is of bounded type, then

for almost every $x \in E$.

$$
\lim _{n \rightarrow \infty} S_{P_{n}}(x)=f(x)
$$

Proof. We may suppose that $E$ is of positive Haar measure, i.e., $m(E)>0$. Fix $0<\epsilon<m(E) / 2$. By hypothesis, $f_{n}(x):=\sigma_{P_{n+1}}(x)$ converges to $f(x)$ for $x \in E$, as $n \rightarrow \infty$. Thus by Egoroff's Theorem, we can choose a measurable subset $E_{0}$ of $E$ such that $f_{n} \rightarrow f$ uniformly on $E_{0}$ and the measure of $E_{0}$ satisfies $m\left(E_{0}\right)>$ $m(E)-\epsilon$.

Let $x \in E_{0}$ be a point which satisfies (3) for every sequence $E_{j}$ which shrinks nicely to $x$. For each fixed positive integer $j$, let $\mathcal{W}_{j}$ represent the collection of indices $\ell$ which satisfy $I_{j+1}(\ell) \subset I_{j}(x)$. If $\ell \in \mathcal{W}_{j}$ and

$$
B_{j}:=I_{j+1}(\ell) \neq A_{j}:=I_{j+1}(x),
$$

let $E_{j}$ represent the union of $A_{j}$ and $B_{j}$. By construction, $E_{j} \subset I_{j}(x)$ and by (2),

$$
m\left(E_{j}\right)=2 m\left(I_{j+1}(x)\right)=\frac{2}{p_{j} P_{j}} \geq \frac{2}{M P_{j}}=\frac{2}{M} \cdot m\left(I_{j}(x)\right)
$$

where $M:=\sup \left\{p_{i}: i \in \mathbf{N}\right\}$. Thus $A_{j}$ and $E_{j}$ both shrink nicely to $x$, as $j \rightarrow \infty$. It follows from (3) that $m\left(E_{0} \cap A_{j}\right) / m\left(A_{j}\right) \rightarrow 1$ and $m\left(E_{0} \cap E_{j}\right) / m\left(E_{j}\right) \rightarrow 1$ as $j \rightarrow \infty$.

Let $n \in \mathbf{N}$ be so large that $m\left(E_{0} \cap A_{n}\right) / m\left(A_{n}\right)<5 / 4$ and $m\left(E_{0} \cap E_{n}\right) / m\left(E_{n}\right)>$ $3 / 4$. Since $B_{n}=E_{n} \backslash A_{n}$ and $m\left(E_{n}\right)=2 m\left(A_{n}\right)=2 m\left(B_{n}\right)$, such a choice for $n$ implies

$$
\begin{aligned}
\frac{m\left(E_{0} \cap B_{n}\right)}{m\left(B_{n}\right)} & =\frac{m\left(E_{0} \cap E_{n}\right)-m\left(E_{0} \cap A_{n}\right)}{m\left(B_{n}\right)} \\
& =\frac{2 m\left(E_{0} \cap E_{n}\right)}{m\left(E_{n}\right)}-\frac{m\left(E_{0} \cap A_{n}\right)}{m\left(A_{n}\right)}>\frac{6}{4}-\frac{5}{4}>0 .
\end{aligned}
$$

In particular, $E_{0} \cap B_{n}$ is uncountable.

We have proved that if $n$ is sufficiently large, for each $\ell \in \mathcal{W}_{n}$ which satisfies $I_{n+1}(\ell) \neq I_{n+1}(x)$, there exists an $x_{n}(\ell) \in E_{0} \cap I_{n+1}(\ell)$. The restriction $I_{n+1}(\ell) \neq$ $I_{n+1}(x)$ is not needed. Indeed, if $\ell$ satisfies $I_{n+1}(\ell)=I_{n+1}(x)$, then $x_{n}(\ell):=x$ also belongs to $E_{0} \cap I_{n+1}(\ell)$. Thus, for $n$ sufficiently large, it is possible to choose points $x_{n}(\ell) \in E_{0} \cap I_{n+1}(\ell)$ for all $\ell \in \mathcal{W}_{n}$. 
We claim that for these points,

$$
\sum_{\ell \in \mathcal{W}_{n}} \sigma_{P_{n+1}}\left(x_{n}(\ell)\right)=\sigma_{P_{n}}(x)+\left(p_{n}-1\right) S_{P_{n}}(x) .
$$

To verify (5), let $y \in G$ and notice by definition that

$$
\begin{aligned}
\sigma_{P_{n+1}}(y) & =\frac{1}{P_{n+1}} \sum_{k=1}^{P_{n+1}} S_{k}(y) \\
& =\frac{1}{p_{n} P_{n}} \sum_{k=1}^{P_{n}} S_{k}(y)+\frac{1}{p_{n} P_{n}} \sum_{k=P_{n}+1}^{P_{n+1}} S_{k}(y) \\
& =\frac{1}{p_{n}} \sigma_{P_{n}}(y)+\frac{1}{p_{n} P_{n}} \sum_{k=P_{n}+1}^{P_{n+1}} S_{k}(y)=: I_{1}(y)+I_{2}(y) .
\end{aligned}
$$

Look at a typical term $T(y):=a_{k} w_{k}(y)$ in one of these sums where $y=x_{n}(\ell)$. If $T$ comes from $I_{1}$, then $k$ is less than $P_{n}$. Since the Vilenkin function $w_{k}$ is constant on $I_{n}(x)$ and $x_{n}(\ell) \in I_{n}(x)$, it is clear that $T\left(x_{n}(\ell)\right)=T(x)$ for all $\ell \in \mathcal{W}_{n}$. Since the number of intervals of the form $I_{n+1}(\ell)$ which are subsets of $I_{n}(x)$ is $p_{n}$, it follows that

$$
\sum_{\ell \in \mathcal{W}_{n}} I_{1}\left(x_{n}(\ell)\right)=\sum_{\ell \in \mathcal{W}_{n}} \frac{1}{p_{n}} \sigma_{P_{n}}(x)=\sigma_{P_{n}}(x) .
$$

On the other hand, suppose that $T$ comes from $I_{2}$ and $P_{n}<k \leq P_{n+1}$. Notice that $\left\{I_{n+1}(\ell): \ell \in \mathcal{W}_{n}\right\}$ contains every subinterval of $I_{n}(x)$, hence $\left\{w_{k}\left(x_{n}(\ell)\right), \ell \in\right.$ $\left.\mathcal{W}_{n}\right\}$ ranges over every $p_{n}$ th root of unity. Since the sum of $p_{n}$ th roots of unity is zero, we have

$$
\sum_{\ell \in \mathcal{W}_{n}} w_{k}\left(x_{n}(\ell)\right)=0
$$

Consequently, adding up the values of $I_{2}$ at $y=x_{n}(\ell)$, as $\ell$ runs over $\mathcal{W}_{n}$, cancels higher order terms and multiplies lower order terms. Indeed, since the number of intervals of the form $I_{n+1}(\ell)$ which are subsets of $I_{n}(x)$ is $p_{n}$ and $P_{n+1}-P_{n}=$ $\left(p_{n}-1\right) P_{n}$, we have

$$
\sum_{\ell \in \mathcal{W}_{n}} \sum_{k=P_{n}+1}^{P_{n+1}} S_{k}\left(x_{n}(\ell)\right)=\sum_{\ell \in \mathcal{W}_{n}} \sum_{k=P_{n}+1}^{P_{n+1}} S_{P_{n}}(x)=p_{n}\left(p_{n}-1\right) P_{n} S_{P_{n}}(x) .
$$

It follows from (6) that

$$
\sum_{\ell \in \mathcal{W}_{n}} \sigma_{P_{n+1}}\left(x_{n}(\ell)\right)=\sigma_{P_{n}}(x)+\left(p_{n}-1\right) S_{P_{n}}(x),
$$

i.e., (5) holds.

Solve (5) for $S_{P_{n}}$ to write

$$
S_{P_{n}}(x)=\frac{1}{p_{n}-1}\left(\sum_{\ell \in \mathcal{W}_{n}} \sigma_{P_{n+1}}\left(x_{n}(\ell)\right)-\sigma_{P_{n}}(x)\right) .
$$

By construction, $x_{n}(\ell)$ belongs to $I_{n}(x)$, hence $x_{n}(\ell) \rightarrow x$, as $n \rightarrow \infty$, for each $\ell \in \mathcal{W}_{n}$. Since the characters $w_{k}$ are continuous on the group, $w_{k}\left(x_{n}(\ell)\right) \rightarrow w_{k}(x)$, as $n \rightarrow \infty$, for each $k \in \mathbf{N}$. But $f$ is the uniform limit of $f_{n}$ on $E_{0}$ and each $f_{n}$ is a Vilenkin polynomial. Since $x_{n}(\ell) \in E_{0}$, it follows that $f\left(x_{n}(\ell)\right) \rightarrow f(x)$ as $n \rightarrow \infty$. 
Let $g$ (respectively, $g_{n}$ ) represent the real part of $f$ (respectively, of $f_{n}$ ). By construction and the observation just made, we can choose $n$ so large that

$$
\operatorname{Re}\left[\sigma_{P_{n+1}}\left(x_{n}(\ell)\right)\right]=g_{n}\left(x_{n}(\ell)\right) \geq g\left(x_{n}(\ell)\right)-\frac{\epsilon}{2} \geq g(x)-\epsilon .
$$

Substituting this estimate back into (7), we obtain

$$
\begin{aligned}
\operatorname{Re}\left(S_{P_{n}}(x)\right) & \geq \frac{1}{p_{n}-1}\left(p_{n} g(x)-p_{n} \epsilon-\operatorname{Re}\left[\sigma_{P_{n}}(x)\right]\right) \\
& \geq \frac{1}{p_{n}-1}\left(p_{n} g(x)-\operatorname{Re}\left[\sigma_{P_{n}}(x)\right]\right)-M \epsilon .
\end{aligned}
$$

Take the limit infimum of this inequality as $n \rightarrow \infty$, apply hypothesis (4), and then let $\epsilon \rightarrow 0$. It follows that

$$
\liminf _{n \rightarrow \infty} \operatorname{Re}\left[S_{P_{n}}(x)\right] \geq g(x)=\operatorname{Re}[f(x)]
$$

for almost every $x \in E_{0}$. Since $\left|E_{0}\right|>|E|-\epsilon$, we can replace $E_{0}$ by $E$. Hence, $\liminf \operatorname{in}_{n \rightarrow \infty} \operatorname{Re}\left[S_{P_{n}}\right] \geq \operatorname{Re}[f]$ almost everywhere on $E$. By repeating the entire argument with the inequalities in (9) reversed, we see that $\lim \sup _{n \rightarrow \infty} \operatorname{Re}\left[S_{P_{n}}\right] \leq$ $\operatorname{Re}[f]$ almost everywhere on $E$. A similar argument works for the imaginary parts. We conclude that $S_{P_{n}} \rightarrow f$ almost everywhere on $E$ as $n \rightarrow \infty$.

\section{REFERENCES}

1. J.E. Daly and K.L. Phillips, A note on $H^{1}$ multipliers for locally compact Vilenkin groups, Canad. Math. Bull. 41 (1998), 392-397. MR 99j:43005

2. W. Rudin, Real and Complex Analysis, 3rd edition, McGraw-Hill, Inc., 1987. MR 88k:00002

3. L.A. Shaginyan, On definite limits and sets of limit functions of Walsh series, Mat. Sbornik 95 (1974), 263-271.

4. N. Ya. Vilenkin, On a class of complete orthonormal systems, Izv. Akad. Nauk. SSSR, Ser. Mat. 11 (1947), 363-400. MR 9:224h

Department of Mathematics, University of Tennessee, Knoxville, Tennessee 37996 